\title{
EVALUATING THE POTENTIAL BENEFITS OF CUCUMBERS FOR IMPROVED HEALTH AND SKIN CARE
}

\author{
H. Murad, M.A. Nyc
}

\begin{abstract}
Epidemiological and nutritional studies indicate that cucumbers, a fruit in the cucurbitaceae family, have numerous benefits internally, externally and even emotionally. As a food, cucumbers offer superior hydration, as they are about $95 \%$ water. They have been used for decades for their anti-inflammatory benefits on skin, soothing properties for digestion, and other therapeutic uses. The following contribution offers an overview of cucumbers, specifically, their use to augment cellular water and address common conditions (i.e.: skin discoloration and aging, cardiovascular and cancerous diseases, bone health, inflammation, and connective tissue disorders).
\end{abstract}

Key words: Skin care, nutraceuticals, cucumbers, water, nutrition, anti-aging.

\section{Introduction}

The importance of diet on health has been welldocumented; deficiencies in key vitamins and minerals can result in serious developmental and metabolic problems. Undoubtedly, the phrase "you are what you eat" has some merit to it: the better you eat, the better you look and feel. Additionally, eating healthy and nutritious foods boosts immune responses and helps fight infections as well as free radicals.

With so many nutritional requirements to take into account, finding the right natural food sources to meet our body's needs can seem like a daunting task. Each month there are new recommendations or food trends regarding eggs, meat, fat, or even water consumption. Suffice to say, ongoing research of the benefits of foods is needed, and one fruit, which deserves more consideration is the cucumber.

Cucumbers, which are fruits and not vegetables, have long been associated with the spa world and topical skin treatment $(1,2)$. Peering through beauty magazines, will no doubt offer several photos of models basking in relaxation with their eye cucumber slices. These pictures are not exclusively based on marketing the idea of luxurious relaxation and soothing spa treatments. Aside from their cooling effect on skin, cucumber slices offer many benefits to the eyes and surrounding tissues through their hydrating properties, which work to reduce

Corresponding Author: Howard Murad, M.D. 2121 Park Place, 1st Floor, El Segundo, CA 90245, Phone: 310.726.3340, E-mail: drmurad@murad.com dehydration, their high levels of vitamin $\mathrm{K}$ that help reduce dark circles, and the lignans they contain for reducing inflammation (3). Additionally, cucumbers have been used to treat wrinkles and sunburns and have been used as a moisturizer and skin brightener by inhibiting tyrosinase (4). The benefits of cucumbers are not just relegated to topical treatment. In fact, abundant research has shown what can happen when the fruit is consumed.

\section{Background}

Cucumbers, which are related to melons, such as watermelon, cantaloupe and honeydew, are a relatively low-calorie food at just about 15 calories per cup, and are about $95 \%$ water. They contain high levels of lignans, vitamin $\mathrm{K}$, cucurbitacins and their derivatives (triterpenoids), flavonoids (apigenin, luteolin, quercetin, and kaempferol), antioxidants such as beta carotene and vitamin $C$, and $B$ vitamins, among other trace elements and minerals $(5,6)$. With such a high level of water content and the added bonus of naturallyoccuring nutrients and trace minerals, cucumbers could be great supplements to drinking water or even serve as an alternative to consuming sports drinks. In fact, the best way to replenish the body and quench thirst is by consuming water through foods (7). This seems somewhat counter-intuitive since we have consistently been taught to drink water. However, choosing foods with high water content offers the cells in our bodies the much-needed hydration they require for basic everyday functioning as well as the vital nutrients to repair and fortify their membranes (8). What has been gleaned 
from scientific literature is that certain phytochemicals, such as the triterpenes that cucumber contains, may offer important cytoprotective capabilities, among other benefits, that may preserve cutaneous barrier function and cellular immunity $(9,10)$. Simply put, cucumbers don't solely hydrate, they provide added elements that your body needs to fortify and regenerate itself. In fact, researchers identified that cucumbers contain Rutin and ascorbic acid oxidase, which function as free radical scavengers, just one mechanistic property that helps to protect against skin damage (11). Furthermore, some research has indicated that cucumbers have known photoprotective activities and provide an SPF value of 0.2 by itself (12). More recent investigations also concluded that topical creams with cucumber extract showed pronounced decrease in melanin and skin sebum, resulting in skin whitening and anti-acne effects (13). Just how the cucumber does this is not clearly understood, but additional investigations into their uses and components can help us understand the mechanisms at work.

\section{Cucumber uses in medicine}

An abundance of research has concentrated on sustainable botanical ingredients as components in neutraceuticals and cosmeceuticals $(3,14,15)$. However, currently there is renewed interest in ethnobotany, and in researching through clinical trials the scientific basis behind cultural and indigenous uses of plants for medicinal uses. The results of these trials have many times stimulated new avenues in research on plants, vegetables and fruit ingredients and their possible uses within topical or supplement products. Along those lines, cucumber folk medicine includes treatment of diarrhea, gonorrhea, diabetes, hypertension and it has been used to detoxify, as an anti-inflammatory, serum lipids regulator, antioxidant, and analgesic (16). While some of these uses remain unproven, there is accumulating research confirming cucumber's phytochemicals as potential chemopreventive and anticancer agents (17-19).

Cellular dehydration, aging, damage and deterioration are human inevitabilities. Much scientific research has been devoted to decoding cellular processes to unlock methods that would halt, stave off or even transform natural, chronological aging $(20,21)$. The conclusions of these studies have offered new thoughts. Seemingly, the premise that, «Before there was medicine, there was food,» comes to mind with a review of the current data on functional botanicals. Indeed, some of the most exciting clinical findings on phytochemicals have produced new knowledge on how to address old problems, such as with botanicals and photoaging reactive oxygen species, or with cardiovascular disease and cancer (22-25).

Cucumbers can and have been used in numerous ways to help augment the diet and hydrate the body, but a more thorough review of the plant's key nutrients and phytochemicals is warranted to offer practical ideas on the fruit's use for cell fortification and to target specific damage whether external, internal or even the result of emotional stress.

\section{Cyto-pathophysiology}

Stress is a threat that is commonly recognized to have a deleterious effect on our health and well-being. Chronic stress resulting from environmental sources or cultural stress can lead to,cell-deteriorating processes such as inflammation and oxidative stress (26). The result of these processes is an overproduction of reactive oxygen species (ROS) and compromised cellular integrity, as ROS-compromised membranes weaken and allow vital, hydrating intracellular water (ICW) to evacuate into the extracellular matrix (27) Additional research is needed to examine this process in more detail, but theoretically, as inflammation accumulates, it signals cytokines and growth factors to allow matrix matalloproteinases (MMPs) to proliferate, which initiates collagen and elastin breakdown-leading to and a major contributor of chronological aging. In patients with compromised tissues (i.e.: dehydrated cells), this process occurs more rapidly as cytology has shown that when cells and connective tissues deteriorate, disorders, diseases and death occur. One study illustrates this process clearly as it shows that the elderly, especially if diseased, display reduced ICW (28). Knowing this, it is conceivable that decreased ICW may cause patients to be more vulnerable to the damaging effects of certain toxins, stress levels, cancers, etc. Addressing water loss and inflammation with cucumber consumption could be an effective way to lower stress and fight against the aging process.

\section{Cucumber in an Alkaline Diet}

As a rule of thumb: the more alkaline the diet, the better. High acid foods can cause cell dehydration and can enhance cellular oxidation and impair immunity. The most hydrating foods are those packed with the highest levels of nutrients and are beneficial to cell health. In general, this includes foods that are anti-inflammatory and as low acid to alkaline-forming as possible. Cucumber is one of the most alkaline foods and because of its triterpenes, it may work well to regulate diseases that involve the immune system (9).

Most low acid to alkaline fruits and vegetables are also anti-inflammatory, like cucumber (29). Cucumber counteracts acidic $\mathrm{pH}$ within the body and specifically in the kidneys, which are tasked with keeping blood $\mathrm{pH}$ within normal levels. Over time, blood $\mathrm{pH}$ naturally becomes more acidic as kidney functions decline with age. Normal blood $\mathrm{pH}$ is between 7.35 and 7.45. When the body is forced to constantly regulate blood $\mathrm{pH}$, this overdrive may cause muscle wasting, bone weakening, hypertension, stroke, cardiovascular disease, and memory and cognition morbidity and mortality from chronic 
diseases. Because of the antioxidants and minerals it contains, cucumber combats all of these situations.

A three-year study showed that an alkaline diet can indeed reduce the speed of muscle wasting that naturally occurs with aging (30). Research also suggests that an alkaline diet can assist with chemotherapy treatments, making the treatments more effective. This is the case with cucumber, which contains lignans that are being studied for treating estrogen-related cancers within the body (31-33). While there are no studies that directly show an alkaline diet to prevent cancer, research is ongoing.

In sum, within an alkaline diet, cucumber may assist cells and connective tissue in retaining water as it works synergistically to enhance systemic, brain, and bone health. Additionally, cucumber may provide the support needed for enhanced enzymatic function, intracellularly, to prevent degradation of tissues and slow cell aging. Presumably, an abundance of alkaline cellular reserves buffers the depletion that occurs when the body constantly readjusts to maintain proper $\mathrm{pH}$ and minimize cellular acidity.

\section{Conclusion}

Cucumbers are literally one of the most versatile fruits as it can be used topically, internally and also for mood stability when modulating stress. Early research shows that its phytochemicals may provide cancer drugenhancing activity while it staves off cardiovascular disease. In addition to its soothing properties and digestive benefits, cucumbers fortify cells so they may retain hydrated and work at the highest levels, and may slow age-related cellular deteriorations. Because of its known therapeutic value, more study on cucumber is warranted, which will likely open even more scientific avenues of study and discussion.

Conflict of interest: The authors declare no conflicts of interest.

Ethical standard: The study was approved by the New England Institutional Review Board.

\section{References}

1. Griere M A. Modern Herb. Tigers Books International London, 1st Ed. 1992;1: 239-241.

2. James AD. The Green Pharmacy. Rodale Press Emmaus, Pennsylvania, 1997;pp. 394-459.

3. Lopes, L., Speretta F., Bentley M. Enhancement of skin penetration of vitamin $\mathrm{K}$ using monoolein-based liquid crystalline systems. European Journal of Pharmaceutical Sciences. 2007; 32(3):209-215.

4. Hooda, R. Antiwrinkle herbal drugs. Journal of Pharmacognosy and
Phytochemistry. 2015; 4(4): 277-281.

5. Mukherjee, P. K., et al. Phytochemical and therapeutic potential of cucumber. Fitoterapia. 2013; 84:227-236.

6. The World's Healthiest Foods. Whfoods.org. Cucumbers. Retrieved from http: / / www.whfoods.com/genpage.php?tname=foodspice\&dbid=42.

7. Murad H, Lange D. The Murad Method. New York: Macmillan, 2003.

8. Murad, H. Inclusive Health: A Professional Compendium. 1st ed. El Segundo, CA: American Academy of Esthetics, 2009.

9. Rios JL. Effects of triterpenes on the immune system. J Ethnopharmacol. 2010; 128(1):1-14.

10. Murad, H. Skin Immunity, The Next Generation of Skin. Les Nouvelles Esthetiques \& Spa. 2008; 7:130-136.

11. Katiyar, S., Saify, K., Singh, S, and Rai, M. Botanical study of skin lightening agents. International Journal of Pharmacognosy. 2014; 1(4): 243-249.

12. Suryawanshi, R.K. et al. Towards an understanding of bacterial metabolites prodigiosin and violacein and their potential for use in commercial sunscreens. International Journal of Cosmetic Science. 2015; 37: 98-107.

13. Akhtar, N. et al. Exploring cucumber extract for skin rejuvenation. African Journal of Biotechnology. 2011. 10(7): 1206-1216.

14. Murad H, Tabibian M. The effect of an oral supplement containing glucosamine, aminoacids, mineral, and antioxidants on cutaneous aging. J Dermatol Treat. 2001; 12:47-51.

15. Baliga M S and Katiyar S K. Chemoprevention of photocarcinogenesis by selected dietary botanicals. Photochem Photobiol Sci. 2006; 5:243-53.

16. Abu-Reidah I, Arráez-Román D, Quirantes-Piné R, Fernández-Arroyo S, Segura-Carretero A, Fernández-Gutiérrez A. HPLC-ESI-Q-TOF-MS for a comprehensive characterization of bioactive phenolic compounds in cucumber whole fruit extract. Food Research International. 2012; 46:108-117.

17. Lee DH, Iwanski GB, and Thoennissen NH. Cucurbitacin: ancient compound shedding new light on cancer treatment. Scientific World Journal. 2010; 10:413-8.

18. Rios JL, Recio MC, Escandell JM, et al. Inhibition of transcription factors by plant-derived compounds and their implications in inflammation and cancer. Curr Pharm Des. 2009; 15(11):1212-37.

19. Thoennissen NH, Iwanski GB and Doan NB. Cucurbitacin b induces apoptosis by inhibition of the JAK/STAT pathway and potentiates antiproliferative effects of gemcitabine on pancreatic cancer cells. Cancer Res. 2009; 69(14):5876-84.

20. Murad H. Thoughts on the Process of Aging. Cosmet Dermatol. 2009; 22 (2).

21. Nagy IZs. The frustrating decades of biological aging theories and the hopes for the future. Anti-Aging Bulletin. 2003; 4(16):22-31.

22. Scalbert A, Williamson G. Dietary intake and bioavailability of polyphenols. J. Nutr. 2000; 130:2073S-2085.

23. Murad H, Jankicevic J. Dietary supplementation as a nutrition intervention strategy to increase intracellular water and phase angle. Orlando, FL. American College of Nutrition 48th Annual Meeting and Conference, 2007.

24. Murad H, Shellow W. Pomegranate extract both orally ingested and topically applied to augment the SPF of sunscreens. Cosmetic Dermatology. 2001;10.

25. Talalay P, Fahey J W, Healy Z R, Wehage S L, Benedict A L, Min C, DinkovaKostova A T. Sulforaphane mobilizes cellular defenses that protect skin against damage by UV radiation. PNAS. 2007; 104:17500-17505.

26. Murad, H. Conquering cultural stress: the ultimate guide to anti-aging and happiness. Wisdom Waters Press: Jan 7, 2015.

27. Nagy IZs. The membrane hypothesis of aging. Boca Raton, FL, CRC Press, 1994: 3-10.

28. Ritz P. Chronic cellular dehydration. J Gerontol. 2001; 56A(6): 349-352.

29. Schwalfenberg GK. The alkaline diet: is there evidence that an alkaline ph diet benefits health? J Environ and Public Health. 2012; 2012:727630.

30. Dawson-Hughes B, Harris SS, and Ceglia L. Alkaline diets favor lean tissue mass in older adults. American Journal of Clinical Nutrition. 2008; 87(3):662665.

31. Webb AL, McCullough ML. Dietary lignans: potential role in cancer prevention. Nutr Cancer. 2005;51(2):117-31.

32. Touillaud, Marina S., et al. Dietary lignan intake and postmenopausal breast cancer risk by estrogen and progesterone receptor status. Journal of the National Cancer Institute. 2007; 99(6):475-486

33. McCann, Susan E., et al. Risk of human ovarian cancer is related to dietary intake of selected nutrients, phytochemicals and food groups. The Journal of nutrition. 2003; 133(6):1937-42. 